\title{
A Meshless Cracking Particles Approach for Ductile Fracture
}

\author{
V. Kumar* and R. Drathi**
}

Erratum to: KSCE Journal of Civil Engineering (2014) 18(1):238-248

DOI 10.1007/s12205-014-0164-4

The original version of this article unfortunately contained a mistake.

Errata:

1. The affiliation of the corresponding author was written incorrectly.

Researcher, Dept. of Aerospace Engineering, Indian Institute of Technology Madras, Chennai 600036, India

Will be corrected as

No 6, 97th St., Vadapalani, Chennai, Tamil Nadu 600078, India 